\title{
Cosmetic Potential of Enzymatic Treated Ginseng Leaf
}

\author{
Hyun-Sun Lee ${ }^{1,2}$, Hyun-Jung Lee ${ }^{1}$, Hye Jin Cho ${ }^{1}$, Sung Sun Park ${ }^{3}$, Jin-Man Kim ${ }^{4}$, and \\ Hyung Joo Suh ${ }^{1^{*}}$ \\ ${ }^{1}$ Department of Food and Nutrition, Korea University, Seoul 136-703, Korea \\ ${ }^{2}$ Institutes of Health and Science, Korea University, Seoul 136-703, Korea \\ ${ }^{3}$ Department of Food and Nutrition, Sungshin Women's University, Seoul 136-742, Korea \\ ${ }^{4}$ Department of Food Science and Biotechnology of Animal Resources, Konkuk University, Seoul 143-701, Korea
}

The objective of this study was to evaluate the potential use of ginseng leaf as a cosmetic material. In this research, we employed enzymatic treated ginseng leaf by using Ultraflo $L$ to improve the recovery of ginsenosides from the ginseng leaf and studied the biological activities and skin safety of the enzymatic treated ginseng leaf for use as a cosmetic material. The total ginsenoside contents of the non-enzymatic treated ginseng leaf (NEGL) and Ultraflo L treated ginseng leaf (UTGL) were 271 and $406 \mathrm{mg} / \mathrm{g}$, respectively. The level of metabolite ginsenosides (sum of Rg2, Rg3, Rg5, Rk1, compound K, Rh1, Rh2, and F2) was higher in UTGL (93.1 mg) compared to NEGL $(62.4 \mathrm{mg})$ in one gram ginseng leaf extract. The increase in amounts of ginsenoside types in UTGL compared to NEGL was generally $140 \%$ to $157 \%$. UTGL exhibited relatively higher 2,2-diphenyl-2-picrylhydrazyl hydrate $\left(\mathrm{IC}_{50}, 2.8 \mathrm{mg} / \mathrm{mL}\right.$ ) and 2,2'-azino-bis(3-ethylbenzothiazoline-6-sulfonic acid) diammonium salt $\left(\mathrm{IC}_{50}, 1.6 \mathrm{mg} / \mathrm{mL}\right)$ radical scavenging activities compared to NEGL $(4.8 \mathrm{mg} / \mathrm{mL}$ and $2.2 \mathrm{mg} / \mathrm{mL})$. The UTGL group showed normalized hydrogen peroxide, lipid peroxidation and visual wrinkling grade induced-UVB exposure. The UTGL did not induce any adverse reactions such as erythema and edema on intact skin sites; however, some guinea pigs treated with UTGL on abraded skin sites showed very slight erythema. The primary irritation index (PII) score of UTGL was 0.05 and it was classified as a practically non-irritating material (PII, 0 to 0.5 ). In skin sensitization tests with guinea pigs, UTGL had a positive rate of skin sensitization at $40 \%$, and the mean evaluation score was 0.4 .

Keywords: Panax ginseng leaves, Enzymatic treatment, Ultraflo L, Cosmeceutical

\section{INTRODUCTION}

Panax ginseng (P. ginseng) C.A. Meyer (Araliaceae) is a valuable herb in East Asia that has also gained popularity in the West due to its pharmacological properties for various diseases including atherosclerosis, liver dysfunction, cerebrovascular disease, hypertension, and post-menopausal disorder [1]. In addition, total crude extract from ginseng included in dermatological formulations was reported to have several beneficial effects on human and animal skin [2-4]. Other studies report that ginsenoside $\mathrm{Rb} 1$ [5] and $\mathrm{Rb} 2$ [3] promote burn wound healing, and topical applications of ginsenoside compound $\mathrm{K}$ might prevent or improve skin deteriorations such as xerosis and wrinkles, partly ascribed to agedependent decreases in human skin [6]. Furthermore, research has shown the protection of red ginseng extract against acute UVB-irradiated skin aging, such as in- (c) This is an Open Access article distributed under the terms of the Creative Commons Attribution Non-Commercial License (http://creativecommons.org/licenses/by-nc/3.0/) which permits unrestricted non-commercial use, distribution, and reproduction in any medium, provided the original work is properly cited.
Received 2 Jul. 2010, Revised 21 Aug. 2010, Accepted 24 Aug. 2010

*Corresponding author

E-mail: suh1960@korea.ac.kr

Tel: +82-2-940-2853, Fax: +82-2-940-2850 
creases in skin thickness and pigmentation and reductions in skin elasticity [7].

Cultivated ginseng is systematically farmed on open land and harvested after a 5 to 6 year cultivation period. Many are aware of the value of ginseng roots, and several studies have been conducted to elucidate the unexplained healing activities of ginseng. Since most studies have focused on ginseng roots, scientists show less interest in ginseng leaves. Yet, it is possible to harvest ginseng leaves every year unlike ginseng roots. In addition, groups of scientists have found that ginseng leaves are rich in polysaccharides, polyphenol, flavonoids, and ginsenosides [8-10]. Shi et al. [11] reported that the content of ginsenoside Re in $P$. ginseng leaves and roothairs is about five times higher than that of $\operatorname{Rg} 1$, but $\operatorname{Re}$ content is lower than that of $\operatorname{Rg} 1$ in the root. They also suggested that $P$. ginseng leaf may be more beneficial than other plant parts. $\operatorname{Rg} 1, \mathrm{Re}$, and $\mathrm{Rb} 1$ are the three main ginsenosides in extracts of $P$. ginseng roots. Ginseng leaf is known to have six major ginsenosides, $R b 1$, $\mathrm{Rb} 2, \mathrm{Rc}, \mathrm{Rd}, \mathrm{Re}$, and $\mathrm{Rg} 1$ [12]. It is generally believed that dammaranne saponins in ginseng leaf play major roles in its antioxidant activity in animal models [13]. In addition, the polyphenols and flavonoids in ginseng leaf exhibit very potent antioxidant activities [9]. Recent studies have employed new technologies to maximize the herbal benefits of ginseng, and have focused on the development of new ginseng health products [14]. Powders and extracts from ginseng roots have been used to make cosmetics [15]. However, products incorporating ginseng roots are typically too costly because ginseng is not only expensive, but also difficult to cultivate. The objective of this study was to evaluate the potential use of ginseng leaf as a cosmetic material. In this research, we employed enzymatic treated ginseng leaf by utilizing Ultraflo L, which has side activities such as cellulose, $\beta$-xylanase and $\alpha$-arabinofuranosidase to improve ginsenoside recovery. We also studied the bioavailibility and skin safety of the enzymatic treated ginseng leaf as a cosmetic material.

\section{MATERIALS AND METHODS}

\section{Chemical material and plant material}

Standard ginsenosides including compound $\mathrm{K}, \mathrm{Rh} 2$, Rk1, Rg5, Rg3, F2, Rd, Rb2, Rc, Rb1, Rg2(s), Rg2(r), Rh1, Rf, Re, and Rg1 were purchased from Ambo Institute (Seoul, Korea). Folin Ciocalteu's phenol reagent, 2,2-diphenyl-2-picrylhydrazyl hydrate (DPPH), and 2,2'-azino-bis(3-ethylbenzothiazoline-6-sulfonic acid) diammonium salt (ABTS) were purchased from Sigma Chemical Co. (St. Louis, MO, USA). The Ultraflo L was purchased from Novozymes A/S (Bagsværd, Denmark). Ultraflo L is a b-glucanase (endo-1,3(4)-), derived from Humicola insolens, and has side activities such as cellulose and b-xylanase. This enzyme apparently contains significant levels of one or more a-arabinofuranosidase side activities [16]. And then, Ultraflo L standardized to contain $45 \mathrm{FBG} / \mathrm{g}$ (fungal beta glucanase units). Cultivated leaves of Korean $P$. ginseng were collected from a good agricultural practice cultivation garden in Ganghwa-do, which is the major ginseng producing area of Korea. The Korean ginseng leaf was freeze-dried, made into a fine powder, and stored at $-70^{\circ} \mathrm{C}$ until further use.

\section{Preparation of Korean ginseng leaf extraction}

The Korean ginseng leaf powder ( $3 \mathrm{~g}$ ) was rehydrated with distilled water (DW, $50 \mathrm{~mL}$ ) and $0.5 \mathrm{wt} \%$ Ultraflo $\mathrm{L}$ was added at $40^{\circ} \mathrm{C}$ for 12 hours to hydrolyze cellulose and pectin in the ginseng leaf [16-18]. The hydrolyzed mixture was extracted twice with $150 \mathrm{~mL}$ of ethanol under reflux in a water bath at $90^{\circ} \mathrm{C}$ for 2 hours. The extract was then centrifuge at $10,000 \times \mathrm{g}$ for 30 minutes. The supernatant was evaporated and then lyophilized. The non-enzymatic treated ginseng leaf $(3 \mathrm{~g} / 50 \mathrm{~mL} \mathrm{DW})$ was incubated without enzyme at $40^{\circ} \mathrm{C}$ for 12 hours and then extracted.

\section{Determination of chemical composition and gin- senoside content of ginseng leaf extracts}

The amount of total carbohydrate was measured by the phenol-sulfuric acid method [19] using glucose as the respective standard. Uronic acid was estimated by the 3-phenylphenol method [20] using galactronic acid as a standard. The total polyphenol and total flavonoid contents of the extracts were determined by employing Folin-Ciocalteu and $p$-dimethylamino-cinnamaldehyde reagents, respectively, using protocols reported elsewhere [21]. The total phenolic compound and flavonoid contents were expressed as gallic acid equivalents and catechin equivalents, respectively.

The levels of 16 major ginsenosides were analyzed using an HPLC-based technique developed by Lee et al. [22]. The utilized HPLC system was a Varian Prostar 200 HPLC system (Varian Inc., Palo Alto, CA, USA) equipped with a quaternary solvent delivery system, an auto-sampler, and UV detector, measuring at $203 \mathrm{~nm}$. The column was a IMtakt Cadenza CD-C18 column (4.6×75 mM; Imtakt Corporation, Kyoto, Japan). The peak identifications were based on retention times and 
comparisons with injected standard samples.

\section{Antioxidant activities of ginseng leaf extracts}

Antioxidant activity was measured by DPPH radical scavenging activity and ABTS radical scavenging activity. The DPPH radical scavenging activity was measured by the method described by Quang et al. [23]. ABTS radical scavenging activity was determined as described by Wang and Xiong [24]. All tests were performed in triplicate. The antioxidant activities of the test samples were expressed as the median effective concentration for radical-scavenging activity $\left(\mathrm{IC}_{50}\right)$, i.e., the amount of tested extract required for a $50 \%$ decrease in absorbance of DPPH radicals or ABTS radicals.

\section{Determination of ginsenoside content of ginseng leaf extracts}

The levels of 16 major ginsenosides were analyzed using an HPLC-based technique developed by Lee et al. [22]. The utilized HPLC system was a Varian Prostar 200 HPLC system equipped with a quaternary solvent delivery system, an auto-sampler, and UV detector, measuring at $203 \mathrm{~nm}$. The column was a IMtakt Cadenza CD-C18 column. The peak identifications were based on retention times and comparisons with injected standard samples.

\section{Cosmetic formulations}

The formulations that were investigated were based on essence types, as described in Table 1. The formulations included supplemented basic ingredients contained in base essence (BsE), 0.04\% adenosine contained in base essence (AdE), 2\% arbutin contained in base es-

Table 1. Composition of four cosmetic formulations

\begin{tabular}{lcccc}
\hline Formulations (\%) & BsE & AdE & ArE & UTGL \\
\hline Deionized water & 61.5 & 62.5 & 59.5 & 51.5 \\
Carbomer (1\%) & 30 & 30 & 30 & 30 \\
Hyaluronic acid & 5 & 5 & 5 & 5 \\
Beta glucan & 3 & 3 & 3 & 3 \\
Triethanol amine & 0.3 & 0.3 & 0.3 & 0.3 \\
Methyl paraban & 0.1 & 0.1 & 0.1 & 0.1 \\
DS-49 & 0.1 & 0.1 & 0.1 & 0.1 \\
Active ingredient & - & 0.04 & 2 & 10 \\
Total & 100 & 100 & 100 & 100 \\
\hline
\end{tabular}

BsE, base essence type cosmetic formulations; AdE, 0.04\% adenosine essence type cosmetic formulation (BsE+adenosine); $\mathrm{ArE}, 2 \%$ arbutin essence type cosmetic formulation (BsE+arbutin); UTGL, $10 \%$ Ultraflo $L$ treated ginseng leaf (BsE+UTGL extracts); DS-49, disodium-2,2'-dihydroxy-4,4'-dimethoxybenzophenone sulphonic acid. sence (ArE), and 10\% Ultraflo L treated ginseng leaf extract (UTGL) contained in base essence.

\section{Topical treatment and UVB irradiation}

UVB irradiation assays were performed on $\mathrm{HRS} / \mathrm{J}$ strain male hairless mice weighing 25 to $30 \mathrm{~g}$ according to the method of Marquele-Oliveira et al. [25], with some modifications. The experimental protocol was reviewed and approved by the Korea University Animal Care Committee (KUACUC-2010-106).

The skin of the mice was irradiated by UVB irradiation using an ultraviolet lamp, a model TL/12RS 40 W Philips. This particular source emits in the range of 270 to $400 \mathrm{~nm}$ with an output peak at $313 \mathrm{~nm}$, and is equipped with UVB and UV detectors. A $100 \mathrm{mg}$ amount of sample was applied 3 times a day for 7 days on the upper side of the animals and then the animals were irradiated after 3 days. During irradiation, the back was irradiated at $5.2075 \times 10^{-4} \mathrm{~W} / \mathrm{cm}^{2}$ at a distance of 40 $\mathrm{cm}$ as measured by a 1,700 radiometer (International Light Inc., Japan). The animals were fed a standard diet and allowed to drink water ad libitum. They were housed in cages with a 12-hour light and 12-hour dark cycle. The animals were irradiated within their cages. The mouse were sorted into a UVB group group (UVB + no essence) as a negative control group, BsE group (UVB+BsE), $\mathrm{AdE}$ group (UVB $+\mathrm{AdE}), \mathrm{ArE}$ group $(\mathrm{UVB}+\mathrm{ArE})$, UTGL group (UVB + ArE), and a No UVB group (No $\mathrm{UVB}+\mathrm{No}$ essence) as a positive control group.

\section{Evaluation of photoprotective effects}

Skin wrinkling in each group was evaluated by blinded investigators using a grading scale (Table 2) based on the experimental model proposed by Bissett et al. [26].

The animals were killed by decapitation 6 hours after the last UVB exposure on 7 days and the full thickness of the dorsal skin was removed. The skin was washed in $0.15 \mathrm{M} \mathrm{NaCl}$ solution, blotted onto filter paper, weighed,

Table 2. Grading scale of mouse skin wrinkling

\begin{tabular}{cl}
\hline Grade & \multicolumn{1}{c}{ Description of skin } \\
\hline 0 & $\begin{array}{l}\text { Numerous fine striations covering back and flanks of body. Fine } \\
\text { striations run length of body (head-to-tail direction) and appear } \\
\text { and disappear with motion }\end{array}$ \\
1 & $\begin{array}{l}\text { All fine striations on back along spine are gone. A few shallow } \\
\text { coarse wrinkles across back (run perpendicular to head-to-tail di- } \\
\text { rection) which appear and disappear with motion }\end{array}$ \\
2 & $\begin{array}{l}\text { All fine striations gone. Some coarse wrinkles across back (run } \\
\text { perpendicular to head-to-tail direction) which are permanent }\end{array}$ \\
3 & $\begin{array}{l}\text { All fine striations gone. Several deep coarse wrinkles across back } \\
\text { (run perpendicular to head-to-tail direction) which are permanent }\end{array}$ \\
4 & Deep-wrinkled, leathery skin with some flesh-coloured lesions
\end{tabular}


and homogenized in $4.5 \mathrm{~mL}$ of potassium phosphate buffer ( $\mathrm{pH}$ 7.0). The homogenates were centrifuged at $13,000 \times \mathrm{g}$ and at $4^{\circ} \mathrm{C}$ for 4 minutes and the supernatant was used to measure levels of reduced glutathione (GSH), hydrogen peroxide, and lipid peroxidation. The GSH level of the dorsal skin was determined by the method of Tietze [27], expressed as the equivalent $\mu \mathrm{m}$ of GSH per $g$ of tissue. Hydrogen peroxide was determined by the method of Marnett and Ji [28], and the concentration of hydrogen peroxide was calculated against a standard curve and expressed as the equivalent $\mu \mathrm{m}$ of hydrogen peroxide per $\mathrm{g}$ of sample. Lipid peroxidation was estimated by measuring thiobarbituric acid reactive substance concentrations according to the method of Quintanilha et al. [29] and using malondialdehyde as a standard.

\section{Skin safety of Ultraflo $L$ treated ginseng leaf}

Skin safety was evaluated according to the guideline for toxicity of functional cosmetics (KFDA Notification 2008-75) and the experimental protocol was reviewed and approved by the Korea University Animal Care Committee (KUACUC-2010-106). Hartley strain guinea pigs weighing 300 to $400 \mathrm{~g}$ were obtained from Nara biotech (Seoul, Korea). They were individually housed in stainless cages.

The primary irritation test was conducted according to the method of Draize et al. [30]. Each animal was clipped free of hair with an electric clipper and shaved with an electric razor 1 day before testing. A $0.1 \mathrm{~mL}$ amount of $10 \%$ UTGL (vehicle: saline) was spread on cotton lint $(1.5 \times 1.5 \mathrm{~cm})$ and then lints were applied to two sites (one site intact, and the other abraded with a sterilized syringe needle). The application sites were covered and wrapped with an elastic adhesive bandage (3M Transpore ${ }^{\circledR}$; $3 \mathrm{M}$ Health Care, St. Paul, MN, USA). Approximately 24, 48, and 72 hours after application, the animals were examined for signs of irritation. The skin reactions were evaluated in accordance with the following Draize method [30]: (1) Erythema and eschar formation: Score 0, no erythema; Score 1, very slight erythema; Score 2, well-defined erythema; Score 3, moderate to severe erythema; Score 4, severe erythema and slight eschar formation, and (2) Edema formation: Score 0, no edema; Score 1, very slight edema; Score 2, slight edema; Score 3, moderate edema; Score 4, severe edema. The primary irritation index (PII), the sum of the scored reactions (both for erythema/eschar and edema formation) for all animals at 72 hour divided by animal number, was calculated following test completion. Body weights were measured on the day before administration and at test termination for all animals.

Skin sensitization was evaluated according to the maximization test method, as described by Magnusson and Kligman [31]. The first stage of the induction was performed as follows. On 0 day (D-0), the first day of the first stage of induction, (i) $0.1 \mathrm{~mL}$ of injection solvent+Freund's complete adjuvant emulsion (1:1); (ii) $0.1 \mathrm{~mL}$ sample; and (iii) $0.1 \mathrm{~mL}$ sample+Freund's complete adjuvant emulsion (1:1) were injected intradermally at the four corners of the clipped and shaved induction area. After 48 hours (D-2), these patches were removed, and the test sites were gently sponged to remove any remaining sample residue. On day D-6, 0.1 $\mathrm{mL}$ of $10 \%$ sodium lauryl sulfate (Sigma Chemical Co.) in petrolatum was applied to this area. In the second stage of induction, day D-7, $0.1 \mathrm{~mL}$ of each tested material was applied to the area and patched for 48 hours. On day D-22, the animals, whose back hair had been clipped and shaved on day 21 , were exposed to a challenge dose. A $0.1 \mathrm{~mL}$ amount of sample was applied occlusively to a challenge area and patched in a similar way as for the inductions. After 24 hours, the patches were removed, and the test sites were gently sponged to remove any remaining sample residue. Skin reactions were evaluated visually 24 and 48 hours after patch removal according to a well-defined sensitization scoring system [32]. Skin reaction: Score 0, no visible changes; Score 1, discrete or patchy erythema; Score 2, moderate and confluent erythema; Score 3, intense erythema and swelling.

\section{Statistical analysis}

All expressed values are the means of triplicate determinations. All statistical analyses were performed using the SPSS ver. 12.0 (SPSS Inc., Chicago, IL, USA). The statistical significance of differences was determined using unpaired Student's $t$-tests and one-way ANOVA, evaluating significant differences at $p$-values $<0.05$. All data are at the $5 \%$ significance level and are reported as means $\pm \mathrm{SD}$.

\section{RESULTS}

\section{Chemical composition and ginsenoside content of ginseng leaf extract}

In a preliminary study, ginseng leaf was treated with 8 enzymes (Celluclast 1.5 L, Cytolase PCL5, Econase CE, Optidex L-400, Pectinex 5XL, Rapidase TF, Ultraflo L, and Viscozyme L) to improve its yield of ginsenosides. The Ultraflo L treated ginseng leaf extract had 
Table 3. Chemical composition of ginseng leaf

\begin{tabular}{lcc}
\hline & NEGL & UTGL \\
\hline Total sugar $(\mathrm{mg} / \mathrm{mg})$ & $0.47 \pm 0.04$ & $0.67 \pm 0.06$ \\
Uronic acid $(\mu \mathrm{g} / \mathrm{mg})$ & $36.21 \pm 1.70$ & $51.23 \pm 0.29$ \\
Polyphenol $(\mu \mathrm{g} / \mathrm{mg})$ & $23.22 \pm 0.44$ & $33.68 \pm 0.13$ \\
Flavonoid $(\mu \mathrm{g} / \mathrm{mg})$ & $0.10 \pm 0.00$ & $0.16 \pm 0.00$ \\
\hline
\end{tabular}

Values are presented as mean \pm SD $(n=3)$.

NEGL, non-enzymatic treated ginseng leaf; UTGL, Ultraflo L treated ginseng leaf.

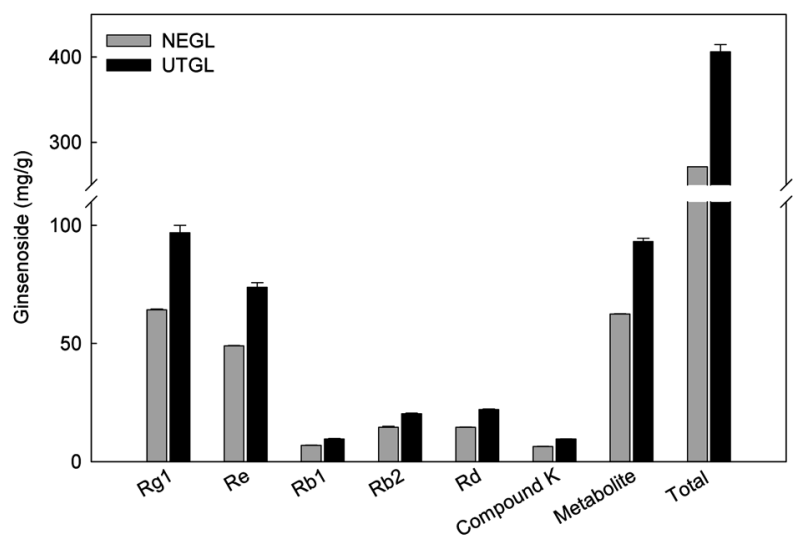

Fig. 1. Gensenoside content of ginseng leaf. The levels of 16 major ginsenosides were analyzed using an HPLC system, IMtakt Cadenza CD-C18 column and UV detector at $203 \mathrm{~nm}$. The peak identifications were based on retention times and comparisons with injected standard samples. Metabolites were the sum of Rg2, Rg3, Rg5, Rk1, compound K, Rh1, Rh2, and F2. Each contents that represents mean \pm SD $(n=3)$. NEGL, non-enzymatic treated ginseng leaf; UTGL, Ultraflo $L$ treated ginseng leaf.

the highest ginsenoside content (data not shown). The chemical composition and ginsenoside compositions of non-enzymatic treated ginseng leaf (NEGL) and UTGL were compared (Table 3 and Fig. 1). Total sugar, uronic acid, and polyphenol, flavonoid contents were higher in UTGL than NEGL. The total ginsenoside contents of NEGL and UTGL in ginseng leaf extract were $271 \mathrm{mg} /$ $\mathrm{g}$ and $406 \mathrm{mg} / \mathrm{g}$, respectively. In addition, the level of metabolite ginsenosides (sum of Rg2, Rg3, Rg5, Rk1, compound $\mathrm{K}, \mathrm{Rh} 1, \mathrm{Rh} 2$, and F2) was higher in UTGL $(93.1 \mathrm{mg} / \mathrm{g})$ than in NEGL $(62.4 \mathrm{mg} / \mathrm{g})$. The increased rate of ginsenoside types in UTGL compared to NEGL was generally $140 \%$ to $157 \%$.

\section{Antioxidant activities}

As shown in Table 3, total polyphenol was higher in UTGL $(33.68 \mu \mathrm{g} / \mathrm{mg})$ than NEGL $(23.22 \mu \mathrm{g} / \mathrm{mg})$. The antioxidant properties of polyphenols and flavonoids are due to their redox properties and the ability to chelate metals and quench singlet oxygen [33]. The radical scavenging activities are summarized in Fig. 2. UTGL exhibited relatively higher DPPH $\left(\mathrm{IC}_{50}, 2.8 \mathrm{mg}\right)$ and ABTS $\left(\mathrm{IC}_{50}, 1.6 \mathrm{mg} / \mathrm{mL}\right)$ radical scavenging activities than UTGL ( $4.8 \mathrm{mg} / \mathrm{mL}$ and $2.2 \mathrm{mg} / \mathrm{mL}$, respectively).

\section{In vivo study to evaluate photoprotective effects}

The UTGL only showed increased yields without conversion of ginsenosides. Therefore, we evaluated the photoprotective effects of an essence type formulation containing 10\% UTGL (total ginsenoside concentration was 4.06\%). We used $0.04 \% \mathrm{AdE}$ and $2 \%$ ArE contained in base essence as positive controls.

UV exposure, particularly UVB, causes the generation of free radicals and related reactive oxygen species (ROS), such as $\mathrm{H}_{2} \mathrm{O}_{2}$. ROS generated as a consequence
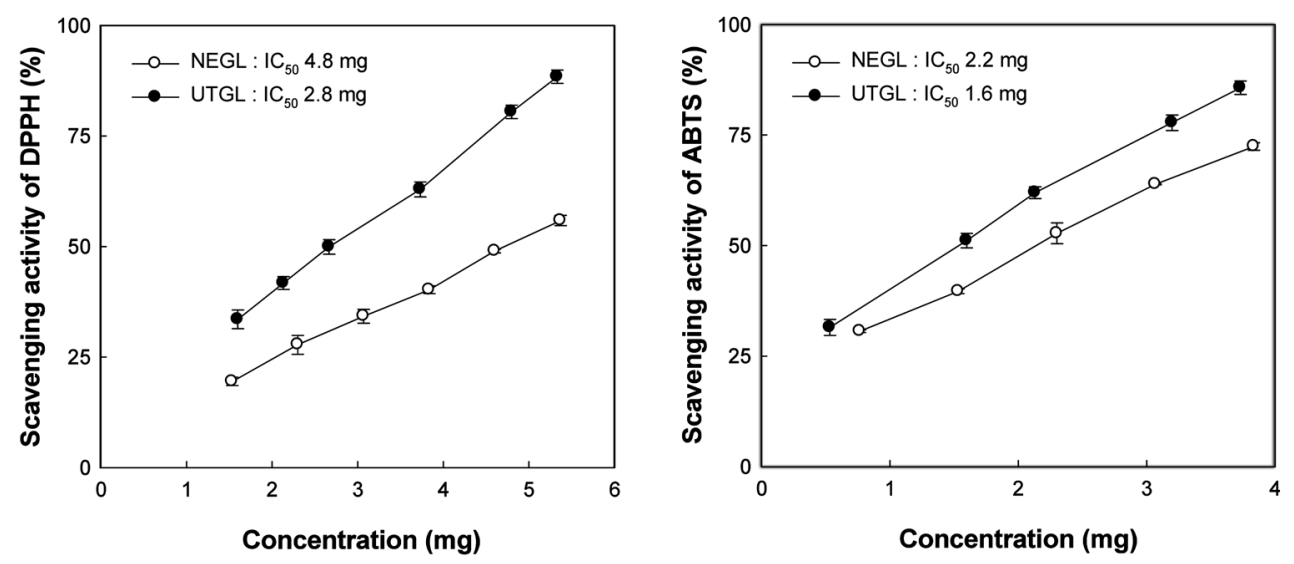

Fig. 2. Antioxidant activities of ginseng leaf. Antioxidant activity was measured by 2,2-diphenyl-2-picrylhydrazyl hydrate (DPPH) radical scavenging activity and 2,2'-azino-bis(3-ethylbenzothiazoline-6-sulfonic acid) diammonium salt (ABTS) radical scavenging activity. All tests were performed in triplicate. The antioxidant activities of the test samples were expressed as the median effective concentration for radical-scavenging activity $\left(\mathrm{EC}_{50}\right)$, i.e., the amount of tested extract required for a $50 \%$ decrease in absorbance of DPPH radicals or ABTS radicals. Each amount represents means \pm SD $(n=3)$. NEGL, non-enzymatic treated ginseng leaf; UTGL, Ultraflo L treated ginseng leaf. 
of UVB exposure produce oxidative stress, intracellular GSH depletion, and increased products of lipid peroxidation. This may be the reason for wrinkle formation in photoaged skin $[34,35]$. As shown in Fig. 3, the base essence group (BsE) had reduced hydrogen peroxide formation and a lower visual wrinkling grade compared to the no cream group since the BsE formulation contained some available compound. However, treatments of active ingredients such as adenoisin or arbutin were significantly more effective than BsE. The UTGL group showed normalized hydrogen peroxide and lipid peroxidation induced-UVB exposure that was similar to the non exposure group. Finally, in terms of visual wrinkling grade, the UTGL group showed a similar tendency to the non-UVB treated control group with anti-wrinkles effects against UVB exposure.

\section{Primary irritation and skin sensitization testing of UTGL}

The skin safety of cosmetic ingredients is more important than their function. Therefore, we evaluated the safety of UTGL using primary irritation and skin sensitization tests. Table 4 shows the results of the primary skin irritation test. The guinea pigs had 10\% UTGL dermally attached for 24 hours. The UTGL did not induce any adverse reactions such as erythema and edema on intact skin sites; however, some guinea pigs treated with UTGL on abraded skin sites showed very slight erythema. The PII score of UTGL was 0.05 and it was classified as a practically non-irritating material (PII, 0
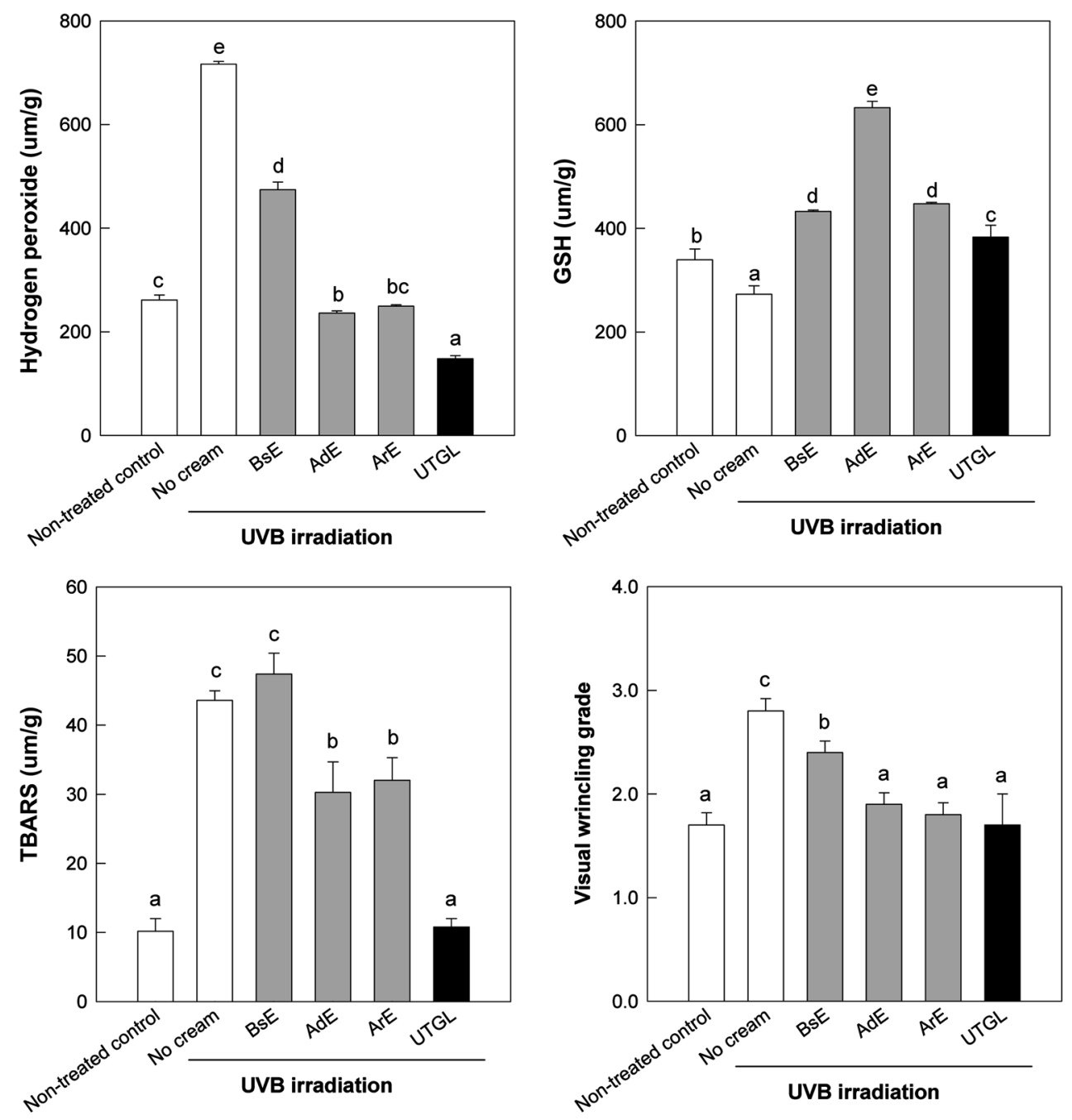

Fig. 3. Effects of cream type formations with added active ingredients on hydrogen peroxide level (A), reduced glutathione level (B), lipid peroxidase level (C), and visual wrinkling grade (D) in UVB-irradiated hairless mice. Different letters at each measurement indicate significant differences among groups $(p<0.05)$. Each bar represents means $\pm \mathrm{SD}(n=5)$. BsE, base essence; AdE, BsE+adenosine; ArE, BsE+arbutin; UTGL, BsE+Ultraflo L treated Korean ginseng leaf extracts; GSH, reduced glutathione; TBARS, thiobarbituric acid reactive substance. 
Table 4. Evaluations of skin irritation by NEGL and UTGL

\begin{tabular}{|c|c|c|c|c|c|c|c|c|c|c|c|c|c|c|c|}
\hline & \multirow{2}{*}{ Skin reaction } & & \multirow[b]{2}{*}{$\mathrm{h}$} & \multicolumn{10}{|c|}{ Score of skin irritation (No. of animal) } & \multirow{2}{*}{$\begin{array}{l}\text { Mean } \\
\text { score }\end{array}$} & \multirow{2}{*}{ PII } \\
\hline & & & & 1 & 2 & 3 & 4 & 5 & 6 & 7 & 8 & 9 & 10 & & \\
\hline \multirow{8}{*}{$\begin{array}{l}\text { Control } \\
\text { (saline) }\end{array}$} & \multirow{4}{*}{$\begin{array}{l}\text { Intact } \\
\text { skin }\end{array}$} & \multirow{2}{*}{ Erythema } & 24 & 0 & 0 & 0 & 0 & 0 & 0 & 0 & 0 & 0 & 0 & 0 & \multirow{8}{*}{0.00} \\
\hline & & & 72 & 0 & 0 & 0 & 0 & 0 & 0 & 0 & 0 & 0 & 0 & 0 & \\
\hline & & \multirow{2}{*}{ Edema } & 24 & 0 & 0 & 0 & 0 & 0 & 0 & 0 & 0 & 0 & 0 & 0 & \\
\hline & & & 72 & 0 & 0 & 0 & 0 & 0 & 0 & 0 & 0 & 0 & 0 & 0 & \\
\hline & \multirow{4}{*}{$\begin{array}{l}\text { Abraded } \\
\text { skin }\end{array}$} & \multirow{2}{*}{ Erythema } & 24 & 0 & 0 & 0 & 0 & 0 & 0 & 0 & 0 & 0 & 0 & 0 & \\
\hline & & & 72 & 0 & 0 & 0 & 0 & 0 & 0 & 0 & 0 & 0 & 0 & 0 & \\
\hline & & \multirow{2}{*}{ Edema } & 24 & 0 & 0 & 0 & 0 & 0 & 0 & 0 & 0 & 0 & 0 & 0 & \\
\hline & & & 72 & 0 & 0 & 0 & 0 & 0 & 0 & 0 & 0 & 0 & 0 & 0 & \\
\hline \multirow{8}{*}{ UTGL } & \multirow{4}{*}{$\begin{array}{l}\text { Intact } \\
\text { skin }\end{array}$} & \multirow{2}{*}{ Erythema } & 24 & 0 & 1 & 0 & 0 & 2 & 0 & 0 & 0 & 0 & 0 & 0.3 & \multirow{8}{*}{0.05} \\
\hline & & & 72 & 0 & 1 & 0 & 0 & 1 & 0 & 0 & 0 & 0 & 0 & 0.2 & \\
\hline & & \multirow{2}{*}{ Edema } & 24 & 0 & 0 & 0 & 0 & 0 & 0 & 0 & 0 & 0 & 0 & 0 & \\
\hline & & & 72 & 0 & 0 & 0 & 0 & 0 & 0 & 0 & 0 & 0 & 0 & 0 & \\
\hline & \multirow{4}{*}{$\begin{array}{l}\text { Abraded } \\
\text { skin }\end{array}$} & \multirow{2}{*}{ Erythema } & 24 & 0 & 0 & 0 & 0 & 0 & 0 & 0 & 0 & 0 & 0 & 0 & \\
\hline & & & 72 & 0 & 0 & 0 & 0 & 0 & 0 & 0 & 0 & 0 & 0 & 0 & \\
\hline & & \multirow{2}{*}{ Edema } & 24 & 0 & 0 & 0 & 0 & 0 & 0 & 0 & 0 & 0 & 0 & 0 & \\
\hline & & & 72 & 0 & 0 & 0 & 0 & 0 & 0 & 0 & 0 & 0 & 0 & 0 & \\
\hline
\end{tabular}

Mean score=total score/number of animals (10).

NEGL, non-enzymatic treated ginseng leaf; UTGL, Ultraflo L treated ginseng leaf; PII, primary irritation index, $\Sigma$ total score/(animal No.×4).

Table 5. Sensitization scores and rates of challenge reactions 72 hours after topical application of UTGL

\begin{tabular}{|c|c|c|c|c|c|c|c|c|c|c|c|}
\hline \multicolumn{3}{|c|}{ Skin reaction } & \multirow[b]{2}{*}{$\mathrm{h}$} & \multicolumn{5}{|c|}{ No. of animal } & \multirow{2}{*}{$\begin{array}{l}\text { Mean } \\
\text { response }\end{array}$} & \multirow{2}{*}{$\begin{array}{l}\text { Sensitization } \\
\text { rate }(\%)\end{array}$} & \multirow{2}{*}{$\begin{array}{l}\text { Evaluation } \\
\text { grade (class) }\end{array}$} \\
\hline Group & Induction & Challenge & & 1 & 2 & 3 & 4 & 5 & & & \\
\hline \multirow{3}{*}{$\begin{array}{l}\text { Negative } \\
\text { control }\end{array}$} & \multirow{3}{*}{ Saline } & \multirow{3}{*}{ Saline } & 24 & 0 & 0 & 0 & 0 & 0 & 0 & & \\
\hline & & & 48 & 0 & 0 & 0 & 0 & 0 & 0 & 0 & I (weak) \\
\hline & & & 72 & 0 & 0 & 0 & 0 & 0 & 0 & & \\
\hline \multirow{3}{*}{$\begin{array}{c}\text { Positive } \\
\text { control }\end{array}$} & \multirow{3}{*}{$\begin{array}{c}1 \% \\
\text { DNCB }\end{array}$} & \multirow{3}{*}{$\begin{array}{c}0.1 \% \\
\text { DNCB }\end{array}$} & 24 & 2 & 3 & 2 & 2 & 1 & 2.0 & & \\
\hline & & & 48 & 3 & 2 & 3 & 3 & 2 & 2.4 & 100 & V (extreme) \\
\hline & & & 72 & 1 & 3 & 3 & 2 & 2 & 2.2 & & \\
\hline \multirow{3}{*}{ UTGL } & \multirow{3}{*}{$\begin{array}{l}10 \% \\
\text { UTGL }\end{array}$} & \multirow{3}{*}{$\begin{array}{c}1 \% \\
\text { UTGL }\end{array}$} & 24 & 1 & 2 & 1 & 1 & 1 & 1.5 & & \\
\hline & & & 48 & 0 & 1 & 0 & 0 & 0 & 0.4 & 40 & III (moderate) \\
\hline & & & 72 & 0 & 1 & 0 & 0 & 1 & 0.4 & & \\
\hline
\end{tabular}

UTGL, Ultrafl o L treated ginseng leaf; DNCB, dinitrochlorobenzene.

to 0.5$)$.

In the skin sensitization test, the guinea pigs were sensitized with intradermal injections of 10\% UTGL for 48 hours. After 1 week, they were treated on the injection site with the same concentration and then challenged with a $1 \%$ solution 2 weeks later, and observed for 24 , 48 , and 72 hours.

Table 5 shows the skin sensitization test results of the guinea pigs. In the negative control group (saline), the positive rate after evocation was $0 \%$ and the mean evaluation score was 0 . In the UTGL group, the positive rate 72 hours after evocation was $40 \%$, and the mean evaluation score was 0.4 . In the positive control dinitrochlorobenzene group, the positive rate was $100 \%$ and the mean evaluation score was 2.2.

\section{DISCUSSION}

P. ginseng has been shown to possess a wide range of biological and pharmacological activities and is used as a general tonic in traditional oriental medicine to increase vitality, health, and longevity, especially in older 
persons. Previous research has reported that ginseng has several beneficial effects on human and animal skin [3-7]. Kim et al. [36] reported that topical applications of total ginseng saponins (10 pg or $100 \mathrm{ng} /$ mouse) and ginsenoside $\mathrm{Rb} 1$ (100 fg, $10 \mathrm{pg}$, or $1 \mathrm{ng} /$ mouse) isolated from red ginseng significantly inhibited increases in skin thickness and wrinkle formation and reduced skin elasticity induced by long-term UVB irradiation. Furthermore, Lee et al. [37] reported that the topical administration of a cream base containing red ginseng (percentage of ginseng saponins was about 3.3\%) for 22 weeks resulted in significantly decreased wrinkling scores, minimal epidermal hyperplasia, slightly increased dermal cellularity, and a lack of cyst proliferation by UV-irradiation. Thus, ginseng root powder and extracts have been used to make cosmetics [15]. However, products containing ginseng root ingredients are too costly because ginseng is not only expensive, but also difficult to cultivate. Ginseng should be harvested after a 5 to 6 year cultivation period. However, it is possible to harvest ginseng leaves every year, unlike ginseng roots.

Traditionally, ginseng leaf has been consumed mostly in the form of tea. Ginseng leaf is rich in polysaccharides, polyphenols, and flavonoids [8-10], and contents of some ginsenosides are higher than in the roots [11]. However, these compounds are tightly linked with carbohydrates such cellulose, pectin, or $\beta$-glucan. We prepared enzymatic treated ginseng leaf using Ultraflo $\mathrm{L}$ to improve ginsenoside recovery. Ultraflo $\mathrm{L}$ is a $\beta$-glucanase (endo-1,3(4)-) and it has side activities such as cellulose, $\beta$-xylanase, and $\alpha$-arabinofuranosidase. The observed increase in polyphenol content in UTGL was accompanied by increases in flavonoid content, antioxidant activities, and ginsenoside contents as compared to NEGL.

The ginsenoside $\mathrm{Rb} 2$ has been reported to improve wound healing, stimulate epidermal cell proliferation, and enhance the expression of protein factors related to cell proliferation, namely, epidermal growth factor and its receptor fibronectin, and its receptor keratin 5/14, as well as collagenase I [3]. In the present study, the ginsenoside $\mathrm{Rb} 1$ and $\mathrm{Rb} 2$ contents of the ginseng leaf treated with Ultraflo L were $9.50 \mathrm{mg} / \mathrm{g}$ and $20.30 \mathrm{mg} / \mathrm{g}$, and in the non-enzymatic treated ginseng leaf they were $6.86 \mathrm{mg} / \mathrm{g}$ and $14.5 \mathrm{mg} / \mathrm{g}$, respectively.

UV exposure causes oxidative stress, intracellular GSH depletion, increased products of lipid peroxidation, and ultimately wrinkle formation of the skin $[34,35]$. The ginseng leaf treated with Ultraflo L $(147.9 \mu \mathrm{m} / \mathrm{g}$ and 10.8 $\mu \mathrm{m} / \mathrm{g}$ ) normalized hydrogen peroxide and lipid peroxidation induced-UVB exposure compared with no cream
(716.2 $\mu \mathrm{m} / \mathrm{g}$ and $43.59 \mu \mathrm{m} / \mathrm{g}$ ). We assume that these effects were due to its antioxidant activity through polyphenols and flavonoids [33,38]. In this study, the polyhenol and flavonoid conents of the ginseng leaf treated with Ultraflo L were $33.7 \mu \mathrm{g} / \mathrm{g}$ and $0.16 \mu \mathrm{g} / \mathrm{g}$, and in the non-enzymatic treated ginseng leaf they were $23.2 \mu \mathrm{g} /$ $\mathrm{g}$ and $0.1 \mu \mathrm{g} / \mathrm{g}$, respectively. UTGL exhibited relatively higher DPPH and ABTS $\left(\mathrm{IC}_{50} ; 2.8 \mathrm{mg}\right.$ and $1.6 \mathrm{mg}$, respectively) radical scavenging activities than UTGL (4.8 $\mathrm{mg}$ and $2.2 \mathrm{mg}$, respectively). Many natural substances such as carotenoids, tocopherols, and polyphenols are able to act as antioxidants and are widely contained in foods and plants. Flavonoids and other polyphenols have the ability to scavenge free radicals, and therefore, delay lipid oxidation [38].

Ginseng may cause a very serious skin reaction called Stevens-Johnson syndrome (SJS). SJS is a life-threatening condition affecting the skin in which cell death causes the epidermis to separate from the dermis. The syndrome is thought to be a hypersensitivity complex affecting the skin and the mucous membranes [39]. In our results, the $10 \%$ ginseng leaf treated with Ultraflo L did not induce any adverse reactions such as erythema and edema on intact skin sites, but some guinea pigs treated with UTGL on abraded skin sites showed very slight erythema. UTGL also showed skin sensitization by using guinea pig maximization testing. It is difficult to determine the reasons between dosage and self-side effects. But $10 \%$ is an extremely high dosage of active ingredient in a cosmetic.

In this study, ginseng leaf treated with Ultraflo L had increased contents of polyphenols and ginsenosides compared to non-enzymatic treated ginseng leaf. The ginseng leaf treated with Ultraflo L had anti-wrinkles effects against photo-damage. However, $10 \%$ ginseng leaf treated with Ultraflo L showed adverse skin safety effects in skin sensitization tests. Therefore, it is considered that ginseng leaves treated with Ultraflo L retain an optimal usage range. From these results, in terms of cost and source availability, ginseng leaves treated with Ultraflo L have advantages over untreated ginseng leaves and have potential as a cosmetic ingredient.

\section{ACKNOWLEDGEMENTS}

This work (grants no. 00038707) was supported by the Business for Cooperative R\&D between the Industry, Academy, and Research Institute funded Korea Small and Medium Business Administration in 2010. 


\section{REFERENCES}

1. Zhang D, Yasuda T, Yu Y, Zheng P, Kawabata T, Ma Y, Okada S. Ginseng extract scavenges hydroxyl radical and protects unsaturated fatty acids from decomposition caused by iron-mediated lipid peroxidation. Free Radic Biol Med 1996;20:145-150.

2. Chang LK, Whitaker DC. The impact of herbal medicines on dermatologic surgery. Dermatol Surg 2001;27:759763.

3. Choi S. Epidermis proliferative effect of the Panax ginseng ginsenoside Rb2. Arch Pharm Res 2002;25:71-76.

4. Keum YS, Park KK, Lee JM, Chun KS, Park JH, Lee SK, Kwon H, Surh YJ. Antioxidant and anti-tumor promoting activities of the methanol extract of heat-processed ginseng. Cancer Lett 2000;150:41-48.

5. Kimura Y, Sumiyoshi M, Kawahira K, Sakanaka M. Effects of ginseng saponins isolated from Red Ginseng roots on burn wound healing in mice. Br J Pharmacol 2006; 148:860-870.

6. Kim S, Kang BY, Cho SY, Sung DS, Chang HK, Yeom MH, Kim DH, Sim YC, Lee YS. Compound K induces expression of hyaluronan synthase 2 gene in transformed human keratinocytes and increases hyaluronan in hairless mouse skin. Biochem Biophys Res Commun 2004;316:348355.

7. Kim YG, Sumiyoshi M, Kawahira K, Sakanaka M, Kimura Y. Effects of red ginseng extract on ultraviolet B-irradiated skin change in C57BL mice. Phytother Res 2008;22:1423-1427.

8. Park HS, Kwak TH, Moon DG, Kim JJ, Cheon J. Development of the novel anti-cancer immunotheraphy for human prostate cancer: in vivo characterization of an immunotropic and anti-cancer activities of the new polysaccharide from the leaves of Panax ginseng C. A. Meyer. Eur Urol 2004;Suppl 3:94.

9. Park SN, Choi SW, Boo YC, Kim CK, Lee TY. Effects of flavonoids of ginseng leaves on erythrocyte membranes against singlet oxygen caused damage. Korean J Ginseng Sci 1990;14:191-199.

10. Xie JT, Mehendale SR, Wang A, Han AH, Wu JA, Osinski J, Yuan CS. American ginseng leaf: ginsenoside analysis and hypoglycemic activity. Pharmacol Res 2004;49:113117.

11. Shi W, Wang Y, Li J, Zhang H, Ding L. Investigation of ginsenosides in different parts and ages of Panax ginseng. Food Chem 2007;102:664-668.

12. Li TSC, Mazza G, Cottrell AC, Gao L. Ginsenosides in roots and leaves of American ginseng. J Agric Food Chem 1996;44:717-720.
13. Attele AS, Wu JA, Yuan CS. Ginseng pharmacology: multiple constituents and multiple actions. Biochem Pharmacol 1999;58:1685-1693.

14. Cho K, Woo HJ, Lee IS, Lee JW, Cho YC, Lee IN, Chae HJ. Optimization of enzymatic pretreatment for the production of fermented ginseng using leaves, stems and roots of ginseng. J Ginseng Res 2010;34:68-75.

15. Wu J, Zhong JJ. Production of ginseng and its bioactive components in plant cell culture: current technological and applied aspects. J Biotechnol 1999;68:89-99.

16. Sorensen HR, Meyer AS, Pedersen S. Enzymatic hydrolysis of water-soluble wheat arabinoxylan. 1. Synergy between alpha-L-arabinofuranosidases, endo-1,4-betaxylanases, and beta-xylosidase activities. Biotechnol Bioeng 2003;81:726-731.

17. Ahn CB, Jeon YJ, Kang DS, Shin TS, Jung BM. Free radical scavenging activity of enzymatic extracts from a brown seaweed Scytosiphon lomentaria by electron spin resonance spectrometry. Food Res Int 2004;37:253-258.

18. Park PJ, Heo SJ, Park EJ, Kim SK, Byun HG, Jeon BT, Jeon YJ. Reactive oxygen scavenging effect of enzymatic extracts from Sargassum thunbergii. J Agric Food Chem 2005;53:6666-6672.

19. Dubois M, Gilles KA, Hamilton JK, Rebers PA, Smith F. Colorimetric method for determination of sugars and related substances. Anal Chem 1956;28:350-356.

20. Blumenkrantz N, Asboe-Hansen G. New method for quantitative determination of uronic acids. Anal Biochem 1973;54:484-489.

21. Arnous A, Makris DP, Kefalas P. Anthocyanin composition and colour characteristics of selected aged wines produced in Greece. J Wine Res 2002;13:23-34.

22. Lee HJ, Jung EY, Lee HS, Kim BG, Kim JH, Yoon TJ, Oh SH, Suh HJ. Bioavailability of fermented korean red ginseng. J Food Sci Nutr 2009;14:201-207.

23. Quang DN, Hashimoto T, Nukada M, Yamamoto I, Tanaka M, Asakawa Y. Antioxidant activity of curtisians I-L from the inedible mushroom Paxillus curtisii. Planta Med 2003;69:1063-1066.

24. Wang LL, Xiong YL. Inhibition of lipid oxidation in cooked beef patties by hydrolyzed potato protein is related to its reducing and radical scavenging ability. J Agric Food Chem 2005;53:9186-9192.

25. Marquele-Oliveira F, Fonseca YM, de Freitas O, Fonseca MJ. Development of topical functionalized formulations added with propolis extract: stability, cutaneous absorption and in vivo studies. Int J Pharm 2007;342:40-48.

26. Bissett DL, Hannon DP, Orr TV. An animal model of solar-aged skin: histological, physical, and visible changes in UV-irradiated hairless mouse skin. Photochem Photo- 
biol 1987;46:367-378.

27. Tietze F. Enzymic method for quantitative determination of nanogram amounts of total and oxidized glutathione: applications to mammalian blood and other tissues. Anal Biochem 1969;27:502-522.

28. Marnett LJ, Ji C. Modulation of oxidant formation in mouse skin in vivo by tumor-promoting phorbol esters. Cancer Res 1994;54:1886s-1889s.

29. Quintanilha AT, Packer L, Davies JM, Racanelli TL, Davies KJ. Membrane effects of vitamin E deficiency: bioenergetic and surface charge density studies of skeletal muscle and liver mitochondria. Ann N Y Acad Sci 1982;393:32-47.

30. Draize JH, Woodard G, Calvery HO. Methods for the study of irritation and toxicity of substances applied topically to the skin and mucous membranes. J Pharmacol Exp Ther 1944;82:377-390.

31. Magnusson B, Kligman AM. The identification of contact allergens by animal assay. The guinea pig maximization test. J Invest Dermatol 1969;52:268-276.

32. Magnusson B, Kligman AM. Allergic contact dermatitis in the guinea pig. Springfield: C.C. Thomas, 1970.
33. Rice-Evans CA, Miller NJ, Paganga G. Structure-antioxidant activity relationships of flavonoids and phenolic acids. Free Radic Biol Med 1996;20:933-956.

34. Fisher GJ, Kang S, Varani J, Bata-Csorgo Z, Wan Y, Datta S, Voorhees JJ. Mechanisms of photoaging and chronological skin aging. Arch Dermatol 2002;138:1462-1470.

35. Gilchrest BA. Skin aging and photoaging: an overview. J Am Acad Dermatol 1989;21:610-613.

36. Kim YG, Sumiyoshi M, Sakanaka M, Kimura Y. Effects of ginseng saponins isolated from red ginseng on ultraviolet B-induced skin aging in hairless mice. Eur J Pharmacol 2009;602:148-156.

37. Lee HJ, Kim JS, Song MS, Seo HS, Moon C, Kim JC, Jo SK, Jang JS, Kim SH. Photoprotective effect of red ginseng against ultraviolet radiation-induced chronic skin damage in the hairless mouse. Phytother Res 2009;23:399-403.

38. Faure M, Lissi E, Torres R, Videla LA. Antioxidant activities of lignans and flavonoids. Phytochemistry 1990;29:37733775.

39. Dega H, Laporte JL, Frances C, Herson S, Chosidow O. Ginseng as a cause for Stevens-Johnson syndrome? Lancet 1996;347:1344. 